\title{
Gesundheit: Das Meikirch-Modell erklärt sie und weist in die Zukunft
}

\section{Johannes Bircher}

Prof. em. Dr. med.

Das Meikirch-Modell definiert einen Menschen als gesund, wenn er in der Lage ist, die Anforderungen des Lebens zu erfüllen. Um dies zu realisieren, verfügt jeder Mensch über Ressourcen, d.h. über ein biologisch gegebenes und ein persönlich erworbenes Potential, die beide dauernd mit der Gesellschaft und der Umwelt in Verbindung stehen. Politik, Forschung und Therapie haben sich bisher vorwiegend auf das biologisch gegebene Potential konzentriert und die anderen Aspekte der Gesundheit mehr im Hintergrund gelassen. Wird das sowohl in der Public Health als auch in der Individualmedizin ausgeglichen, darf erwartet werden, dass sich die Gesundheit der Menschen deutlich verbessert und sich die Gesundheitskosten entsprechend vermindern.

Bitte beachten Sie zu

diesem Thema auch den nachfolgenden Artikel von Jann Schwarzenbach.
Gesundheitssysteme in der entwickelten Welt sind
heute in der Krise. Die Kosten, ausgedrückt als Teil
des Bruttoinlandprodukts, steigen kontinuierlich und
werden immer schwieriger zu finanzieren. Als Reak-
tion darauf entstand eine fortlaufend umfangreichere
Bürokratie, so dass Ärzte und Pflegende einen immer
höheren Teil ihrer Arbeitszeit hinter dem Computer
verbringen müssen. Die Digitalisierung nimmt fort-
laufend zu, und irgendwie stimmen die organisatori-
schen Anforderungen nicht mehr mit den Bedingun-
gen einer erfüllenden Berufsausübung überein. Diese
Trends scheinen sich in der Zukunft weiter zu verstär-
ken. Eine solche Entwicklung steuert allmählich auf

\section{Résumé}

Selon le modèle de Meikirch, une personne est en bonne santé lorsqu'elle est en mesure de répondre aux exigences de la vie. Pour ce faire, chaque être humain dispose de ressources, c'est-à-dire d'un potentiel biologique inné et d'un potentiel personnel acquis, qui sont tous deux en relation permanente avec la société et l'environnement. Ainsi, dans le modèle de Meikirch, la santé correspond à un système adaptatif complexe qui suit ses propres règles dont il faut tenir compte dans les soins de santé. Jusqu'à présent, la politique, la recherche et la thérapie se sont principalement concentrées sur le potentiel biologique inné et ont laissé les autres aspects de la santé au second plan. Si ce déséquilibre est compensé tant dans la santé publique que dans la médecine individuelle, on peut s'attendre à ce que la santé des gens s'améliore considérablement et à ce que les coûts de santé diminuent en conséquence. eine grössere Umstellung zu, die allerdings gegenwärtig noch nicht konkret in Sicht ist.

\section{Bottom-up-Management erforderlich}

Das Gesundheitssystem ist ein komplexes adaptives System [1]. Solche Systeme widersetzen sich einer Steuerung von aussen. Deshalb ist Top-down-Management, wie es heute im Gesundheitswesen betrieben wird, grundsätzlich ungeeignet. Komplexe adaptive Systeme brauchen eine Bottom-up-Organisation. Diese Art von Betriebsführung ist zwar noch relativ neu, doch in der Wirtschaft existiert sie schon an vielen Orten und ist nach mehreren Kriterien wesentlich erfolgreicher als das klassische Top-down-Management. So ist zum Beispiel in Holland eine Spitex entstanden, die nach den neuen Prinzipien geführt wird. Sie ist effektiver und kostengünstiger als die früheren Arbeitsweisen [2]. Analoge Verfahren sind grundsätzlich für das ganze Gesundheitswesen möglich, werden aber noch kaum realisiert. Eine wichtige Voraussetzung für Bottom-upManagement besteht darin, dass das Ziel aller Aktivitäten, d.h. die Verbesserung der Gesundheit und damit das Wesen der Gesundheit, klar ausgedrückt werden kann. Ein bedeutender Fortschritt auf dem Weg zu einer Definition der Gesundheit wurde 2011 an einer Konferenz internationaler Gesundheitsexperten in Holland erreicht und folgendermassen formuliert: «Gesundheit ist die Fähigkeit, sich anzupassen und sich 


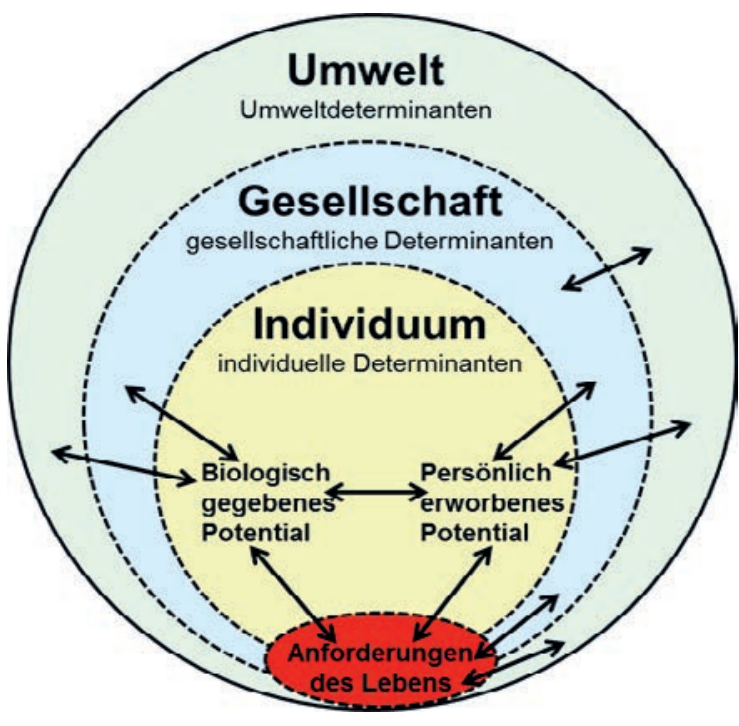

Abbildung 1: Schematische Darstellung des MeikirchModells. Es zeigt die fünf Komponenten und die zehn komplexen Interaktionen, die alle in beiden Richtungen wirken (Doppelpfeile). Mehr Details: www.meikirch-modell.ch. (Aus dem Englischen übersetzt von [6])

selber zu managen» [3]. Dieser Beitrag hat grosse Beachtung erfahren. Doch schon 2005 entstanden die Anfänge des Meikirch-Modells, fokussierten damals aber nur auf das Individuum [4]. In Zusammenarbeit mit Shyama Kuruvilla wurde es 2014 um die Public Health erweitert [5]. Als Resultat entstand ein neues Paradigma, dem das Potential innewohnt, eine Bottom-upErneuerung des Gesundheitssystems ermöglichen und begleiten zu können (www.meikirch-modell.ch). Das Ziel des vorliegenden Artikels über das Meikirch-Modell besteht darin, das gegenwärtige Verständnis für Gesundheit zusammenzufassen.

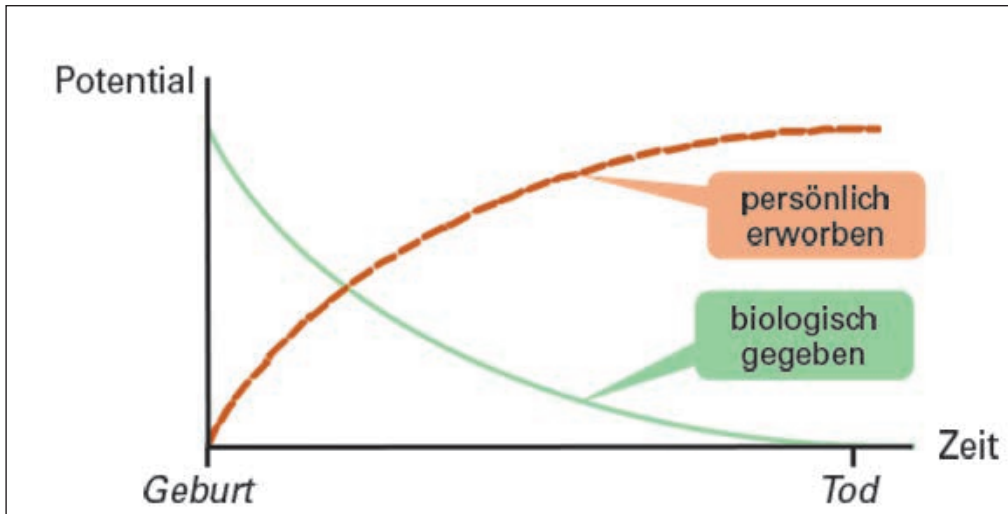

Abbildung 2: Entwicklung der Potentiale während des Lebenslaufs. Wenn z.B. im Alter das BGP gering wird, kann ein gut entwickeltes PEP für viele Defekte kompensieren. Das PEP fällt aber ab, wenn ein Mensch in Drogen gerät, Alkoholiker wird oder eine Psychose erleidet. (Translated by permission from Springer [4])

\section{Erklärung des Meikirch-Modells}

1. Die Abgrenzung zwischen Gesundheit und Krankheit ist von Natur aus unscharf. Es ist aber klar, dass jeder gesunde Mensch in der Lage sein muss, die Anforderungen des Lebens zu erfüllen. Dazu gehören beim Menschen die Erhaltung seines biologischen Lebens, das Arbeiten an persönlicher Erfüllung und die gesellschaftliche Integration (Abb. 1). Kann er es, so ist er gesund, kann er es nicht, so besteht Krankheit. Ein kleines Karzinom irgendwo im Körper wird als «pathologisch» oder krankhaft bezeichnet und gefährdet die längerfristige Zukunft eines Menschen. Der Befund an sich macht ihn aber noch nicht krank. Die meisten Leute mit Kopf- oder Rückenschmerzen sagen, sie seien gesund. Das bedeutet, dass Gesundheit weder mit Abwesenheit von Pathologie noch mit Beschwerdefreiheit gleichzusetzen ist.

2. Doch woher nimmt ein Mensch die Ressourcen, mit denen er die Anforderungen des Lebens erfüllen kann? Zu diesem Zweck verfügt er über ein biologisch gegebenes Potential (BGP) und ein persönlich erworbenes Potential (PEP). Diese beiden Quellen von Energie müssen aber nicht nur in der Gegenwart, sondern auch in der kurzfristigen und langfristigen Zukunft vorhanden sein. Deshalb werden sie als Potentiale bezeichnet.

Das BGP ist ein Geschenk, das jeder Mensch bei der Geburt erhält. Unmittelbar nach der Geburt ist es am grössten, vermindert sich aber im Laufe der Zeit und erreicht den Nullpunkt zum Zeitpunkt des Todes (Abb. 2). Es wird zum Beispiel durch eine gesunde Lebensweise und körperliches Training unterstützt oder durch Übergewicht und Konsum von Drogen vermindert. Jede körperliche Krankheit vermindert das BGP vorübergehend oder dauernd.

3. Das PEP ist bei der Geburt zwar noch klein, aber dennoch schon Ausdruck der Persönlichkeit des Neugeborenen. Es vergrössert sich zunächst rasch, dann immer langsamer. Es kann aber während des ganzen Lebens vermehrt werden, wenn ein Mensch daran arbeitet. Zum persönlich erworbenen Potential gehören alles Gelernte, die Lebensführung, die Persönlichkeit sowie die innere Verarbeitung von Problemen und Schicksalsschlägen. Das persönlich erworbene Potential kann auch einen Teil der Verluste an BGP kompensieren. Ein herausragendes Beispiel ist die Querschnittslähmung. Solche Menschen können den Umgang mit ihrer schwierigen neuen Situation lernen und sogar wieder beruflich tätig werden. Sobald sie den Anforderungen des Lebens erneut genügen, sind sie gemäss MeikirchModell wieder als gesund zu betrachten. 


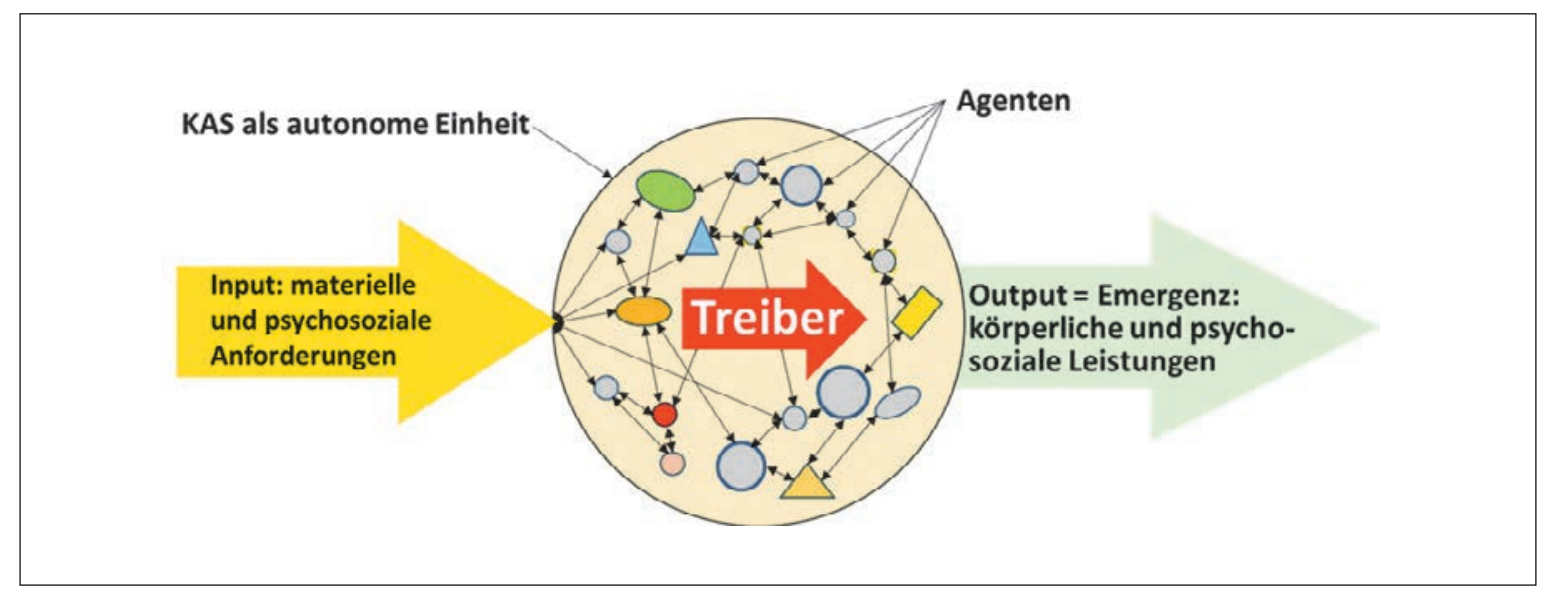

Abbildung 3: Menschliche Gesundheit als komplexes adaptives System. (Abbildung neu)

4. Jeder Mensch lebt im Kontakt mit seinem sozialen Umfeld. Als Säugling wird er von der Mutter gesäugt und betreut. Oft ist ein Vater dabei, und es gibt Geschwister. Später kommen Kindergarten, Schule und Berufsausbildung dazu. So entwickelt sich für die Heranwachsenden auch das soziale Umfeld fortlaufend bis ins Erwachsenenalter, ins Berufsleben und in das eigene Familienleben etc. Auch in jeder weiteren Lebensphase wird der Mensch durch sein soziales Umfeld geprägt. Er prägt aber auch seinerseits dieses Umfeld. Die Kultur der sozialen Beziehungen sowie Frieden und Krieg, Reichtum und Armut hinterlassen ihre Spuren.

5. Als äusserste Schicht des Meikirch-Modells ist jeder Mensch von der natürlichen Umwelt umgeben. Sie enthält die Lebensgrundlagen, aber auch Gefahren und Schwierigkeiten. Heute wird die Umwelt durch den Menschen beschädigt, und er beginnt an den Folgen zu leiden. Beispiele sind Erderwärmung und Plastikabfälle im Meer. Solche Teufelskreise gibt es leider schon viele.

Die fünf Komponenten des Meikirch-Modells sind damit beschrieben. Entscheidend ist festzustellen, dass zwischen allen Komponenten komplexe Austauschprozesse stattfinden, die immer in beiden Richtungen funktionieren (Doppelpfeile in Abb. 1). Es sind deren zehn. Ein typisches Beispiel ist die Beziehung zwischen den beiden Potentialen. Sie kann mit der Beziehung zwischen einem Reiter und seinem Pferd verglichen werden. Wenn der Reiter will, dass ihm sein Pferd gut dient, muss er für das Pferd gut sorgen. Es braucht Futter, Wasser, einen Unterstand für den Schlaf, Sauberkeit, Hufeisen und Bewegung. Eine gute Pferd-Reiter-Beziehung muss auf Gegenseitigkeit stetig und re- spektvoll gelebt werden. Entsprechend dazu muss sich das PEP eines Menschen mit seinem BGP austauschen und dafür Verantwortung übernehmen.

\section{Gesundheit als komplexes adaptives System}

Die vorliegende Kombination von fünf Komponenten und zehn Interaktionen kann als komplexes adaptives System (KAS) verstanden werden [1]. Solche Systeme sind nicht leicht $\mathrm{zu}$ beschreiben. In unserem Zusammenhang haben sie aber eine Anzahl von charakteristischen Eigenschaften, die für den Kontakt mit Menschen, für die Betreuung von Patienten und für die Organisation der Krankenversorgung wichtig sind. Ich möchte hier nur wenige besonders relevante Aspekte hervorheben. Ein komplexes adaptives System ist eine autonome Einheit, die ein Netzwerk von Einzelteilen enthält, die als Agenten bezeichnet werden und sich fortlaufend untereinander austauschen (Abb. 3). Ihre Interaktionen sind entweder einfach (linear) oder komplex (nichtlinear). Die Agenten sind von ihrer "Aussenwelt» abgegrenzt, stehen aber mit ihr fortlaufend im Austausch. So ist bei einem Patienten der Input z.B. materiell oder persönlich (psychosozial). Die produktive Gesamtleistung des KAS wird als Emergenz bezeichnet und umfasst z.B. die geordneten körperlichen und psychosozialen Leistungen der Person. Dank dieser Anordnung funktioniert ein KAS immer als Gesamtheit und selbstbestimmt.

Die Organisation eines KAS schwankt zwischen Stabilität und Labilität. Deshalb ist das KAS in der Lage, sich innerhalb gewisser Grenzen fortlaufend an Veränderungen der Umwelt anzupassen und sich autonom weiterzuentwickeln. Werden aber gewisse Grenzen über- 
schritten, gerät das KAS in eine Krise. Das ist der Fall, wenn es von aussen oder innen starken Einwirkungen ausgesetzt ist. Beim Menschen entstehen Krisen z.B. in der Pubertät, nach einem psychischen Schock, einem Unfall oder bei einem Krankheitserreger. In der Krise versucht das KAS zunächst, die fremden Einflüsse zu kompensieren und zu reparieren, geht aber sobald wie möglich wieder in seinen Vorzustand zurück. Auf alle Fälle widersteht es äusseren Einflüssen so gut und so lang wie nur möglich. Menschen können auch auf autoritär angeordnete Massnahmen mit einer Krise reagieren. Deshalb sind sie oft wirkungslos. Als Alternative gibt es auch echte Anpassungsreaktionen, die zu einer Entwicklung des KAS führen. Die innere Kraft, die ein KAS antreibt, ist der sogenannte Treiber. Er resultiert zum Teil aus der Biologie, d.h. aus Hunger, Durst und anderen Notwendigkeiten des Lebens. Dazu gehören aber in jedem Individuum auch das Bedürfnis nach Lust (Hedonismus), nach Lebenssinn (Eudaimonie) und viele andere Motive. Die Summe der aufgezählten Eigenschaften eines KAS erklärt, dass sich ein Mensch von der Geburt bis zum Tod entwickeln kann und dass bei dieser Entwicklung auch Schwierigkeiten und Krisen auftreten können. Diese werden nicht immer erfolgreich überwunden. Sie können auch Ursachen von Symptomen und Krankheiten sein und müssen zum Teil therapeutisch angegangen werden. Wenn früher die Gesundheit nur aus persönlicher Erfahrung und intuitiv verstanden werden konnte, steht jetzt mit dem Meikirch-Modell ein Konzept von Gesundheit zur Verfügung, das wissenschaftlich begründet, analytisch zerlegbar und rational nachvollziehbar ist (Tab. 1).

\begin{tabular}{|c|c|c|}
\hline Denkweise & $\begin{array}{c}\text { Erkenntnis- } \\
\text { Methode }\end{array}$ & $\begin{array}{c}\text { Nachvollzieh- } \\
\text { barkeit }\end{array}$ \\
\hline $\begin{array}{c}\text { Vor- } \\
\text { wissenschaftlich }\end{array}$ & $\begin{array}{c}\text { Persönliche } \\
\text { Erfahrung }\end{array}$ & intuitiv \\
\hline $\begin{array}{l}\text { Wissenschaftlich: } \\
\text { Meikirch-Modell }\end{array}$ & $\begin{array}{c}\text { Wissenschaftliche } \\
\text { Begründung }\end{array}$ & rational \\
\hline
\end{tabular}

Tabelle 1: Wissenschaftstheoretische Betrachtung der Gesundheit ohne und mit dem Meikirch-Modell. (Tabelle neu)

\section{Konsequenzen für die Medizin}

Das neue Verständnis der Gesundheit im MeikirchModell lässt sich nun vergleichen mit der gegenwärtigen Praxis der Medizin und der Public Health. Daraus ergeben sich interessante Konsequenzen: Wenn die Medizin das PEP und seine Entwicklung als wesentli- chen Bestandteil der Gesundheit entdecken und die Public Health sich schon bei Kindern für eine neue Gesundheitskultur einsetzen würde, dürfte man mit mehr Gesundheit und wesentlich geringeren Kosten rechnen.

\section{Biologisch gegebenes Potential (BGP)}

Die somatische Medizin hat sich darauf konzentriert, Störungen des BGP so gut wie möglich mit Medikamenten, Bestrahlungen, Operationen und Rehabilitationsmassnahmen zu therapieren. Dabei hat sie Grosses erreicht, denn viele Krankheiten, die früher nicht behandelt werden konnten, sind heute einer Therapie zugänglich. Auch arbeitet die medizinische Forschung daran, weitere innovative Therapien zu entwickeln. Neue Medikamente werden zwar immer teurer, doch verschiedene therapeutische Möglichkeiten haben sich in den letzten Jahren entscheidend verbessert. Zurzeit kommt z.B. als neue Idee die digitale Selbstvermessung (Self-Tracking) dazu. Sie kann bis zum "quantified Self» vorangetrieben werden. Wie gross der Nutzen sein wird, ist noch unklar. Auch die personalisierte Medizin ist ein interessanter neuer Ansatz. Sie will sich an einer grossen Summe von individuellen, genetischen Merkmalen kranker Menschen orientieren, um Prädiktion und Therapie möglichst passgenau auf den einzelnen Patienten auszurichten. Auch diese Forschungsrichtung ist noch in Entwicklung. Doch schon ohne die beiden gegenwärtigen Grossprojekte verfügt die Medizin heute über viele äusserst wertvolle Möglichkeiten, das BGP von Patienten zu schützen.

\section{Persönlich erworbenes Potential (PEP)}

Das PEP ist für die wissenschaftlich orientierte Medizin leider immer noch ein relativ «blinder Fleck», der gerne links liegengelassen wird. Sogar in der Psychiatrie sind heute medikamentöse Therapien im Vordergrund, d.h., psychische Störungen und Krankheiten werden vordergründig wie somatische Krankheiten einfach mit Medikamenten behandelt. Das ist nicht grundsätzlich falsch, denn die grossen antipsychotischen Medikamente sind für viele schwerkranke Menschen eine entscheidende Hilfe. Doch die Möglichkeiten, ein KAS, dessen Entwicklung aus irgendeinem Grund in einer Krise blockiert wurde, therapeutisch anzugehen - z.B. mit den verschiedenen Formen von Psychotherapie -, werden leider vernachlässigt. Das ist verständlich, wenn man bedenkt, wie schwierig es sein kann, ein KAS wieder in Bewegung zu versetzen. Es werden heute zwar viele Techniken angeboten, um durch eigene Aktivitäten psychisch besser ins Gleichgewicht zu kommen und sich als Person entwickeln zu können. Zahlreiche von ihnen sind aber wissenschaft- 
lich nicht genügend gut untersucht, um sie allgemein zu empfehlen. Hier gibt das Konzept des KAS interessante neue Möglichkeiten, die auf weitere Entwicklungen warten. Eine Verbesserung von Methoden der Selbstwirksamkeit könnte auch viele somatische Störungen lindern oder verhindern.

Wenn es um die Entwicklung des PEP geht, besteht die Schwierigkeit darin, dass hier die persönliche und die soziale Verantwortung verschwimmen und auch schwierig zu trennen sind. Es wäre wichtig, besser zu verstehen, wie die beiden Verantwortungsbereiche bei verschiedenen Krankheiten zusammenwirken und was sie für die Krankenversicherung bedeuten. Persönliche Entwicklung wird allgemein als ein Bereich der Eigenverantwortung gesehen, obwohl die Gesellschaft keine Methoden anbietet, sie zu unterstützen. Im Hinblick auf die überteuerte Gesundheitsversorgung ist es problematisch, solche Therapien in die Krankenversicherung aufzunehmen. Es ist aber auch nicht richtig, sie ohne Unterstützung jedem Einzelnen zu überlassen. Das Problem der Eigenverantwortung für die Gesundheit ist im ganzen Gesundheitswesen noch ungenügend durchdacht und erfordert dringend eine vertiefte Forschung.

\section{Konsequenzen für die Public Health}

Die Public Health leistet schon sehr viel, ist aber an Grenzen gestossen, die sie mit grösseren Anstrengungen kaum wird überwinden können. Das hängt damit zusammen, dass Anregungen und Informationen zur Vermeidung von Krankheitsrisiken von aussen - d.h. Top-down - an die Menschen herangetragen und entsprechend abgewehrt werden. Sie erreichen deshalb den Treiber ihres KAS für Gesundheit nicht. Neue Möglichkeiten sind dringend zu erforschen. Für den Erfolg der Gesundheitsförderung wird es ratsam sein, auf die
Entwicklung des PEP zu fokussieren und das am besten mit einem Ansatz über den Treiber des KAS zu kombinieren. Wenn er beeinflusst werden soll, so ist die Gesundheitsförderung schon im Kindesalter zu beginnen, d.h. zu einem Zeitpunkt, an dem das KAS und der Treiber sich in einer frühen Entwicklung befinden. Von der ersten Klasse bis zum Ende der Ausbildung sind deshalb die Voraussetzungen gut, eine realistische Vorstellung von Gesundheit zu verinnerlichen. Es sollte eine neue Gesundheitskultur vermittelt werden. Diese beruht zwar auch auf Wissen über die Gesundheit, entscheidend ist aber, dass die Kinder zur richtigen Zeit erleben, was der Treiber ist, wie er sich in ihnen entwickelt und wie sie sich selber für ein sinnvolles Leben einsetzen können. Zu diesem Zweck ist Ausbildung in Selbstführung von besonderer Wichtigkeit. Kinder und Jugendliche sollten schon früh erfahren, wie sie schlechten Gefühlen selber vorbeugen und sich mit ihnen positiv auseinandersetzen können. Umgang mit Symbolen, Meditationstechniken, gewaltfreier Kommunikation und weiteren analogen Verfahren ist in diesem Zusammenhang auch nützlich.

Literatur

1 Sturmberg JP. Health System Redesign. 2018. DOI:10.1007/978-3319-64605-3.

2 Laloux F. Reinventing Organizations. München: Franz Vahlen; 2017.

3 Huber M, Knottnerus JA, Green L, et al. How should we define health? Br Med J. 2011;343:d4163.

4 Bircher J. Towards a dynamic definition of health and disease. Med Heal Care Philos. 2005;8. DOI:10.1007/s11019-005-0538-y.

5 Bircher J, Kuruvilla S. Defining health by addressing individual, social, and environmental determinants: new opportunities for health care and public health. J Public Health Policy. 2014;35:363-86.

6 Bircher J, Hahn EG. Understanding the nature of health: New perspectives for medicine and public health. Improved wellbeing at lower costs. F1000Research. 2016;5:167. 